\title{
Introduction to the Special Issue on Genetic Counseling: A Global Perspective
}

\author{
Beverly M. Yashar • Madelyn Peterson
}

Received: 2 September 2013 / Accepted: 9 September 2013/Published online: 11 October 2013

(C) National Society of Genetic Counselors, Inc. 2013

\begin{abstract}
This special issue of the Journal of Genetic Counseling is focused on the global genetic counselling community and includes commentaries, original research and multiple brief reports describing both established formal genetic counselling services and training programs as well as services and programs that are currently in development.
\end{abstract}

Keywords Global perspectives · Ethnocultural viewpoints · Genetic counselling education $\cdot$ Clinical genetic counselling services

The advent of genetic counseling, as an active service and a health care profession, will always be recognized as primarily emanating from the United States. The historical roots are grounded in Michigan in 1940 and Minnesota in 1941 with the establishment of the first dedicated modern genetics clinics (McKusick 1997). The term "genetic counseling" was coined by Sheldon Reed (a PhD trained genetic scientist and director of the Dight Institute of Human Genetics at the University of Minnesota starting in 1947) who explained it as "a kind of genetic social work without eugenic connotations" (Reed 1975, p.335). The establishment of the first training program for non-medically trained genetic counselors in 1969 at Sarah Lawrence College in the United States formalized the profession (Resta 2006). From these roots, genetic counseling practitioners in the United States expanded their scope of practice by relying on a combination of "educational experimentation, audacious expectations and fortuitous timing" (Stern 2009, p.1).

B. M. Yashar $(\bowtie)$

Department of Human Genetics, University of Michigan,

Ann Arbor, MI, USA

e-mail: Yashar@med.umich.edu

M. Peterson

Griffith University, Nathan, QLD, Australia
The current practice of genetic counselling is focused on helping people understand and adapt to the medical, psychological, and familial implications of genetic contributions to disease, using a process that integrates (1) the interpretation of family and medical histories to assess the chance of disease occurrence or recurrence; (2) education about inheritance, testing, management, prevention, resources, and research; and (3) counseling to promote informed choices and adaptation to the risk or condition (Resta 2006). The increasing importance of genetics to so many aspects of health care, and the critical ways in which genetic counseling positively reinforces and promotes knowledge acquisition and understanding, informed autonomous decision making, and support for individuals and families affected by genetic conditions, made it inevitable that the practice and the dedicated profession would extend globally. The 30 papers in this special edition are proof of this growth.

Over the last decade, there have been significant attempts to connect the global community of professionals working in genetic counseling. Many genetic counselors from outside the United States have joined the National Society of Genetic Counselors (NSGC) to become part of the world's largest and most proactive professional society for genetic counselors. Significantly, in 2006 a Jane Engelberg Memorial Fellowship awarded to Janice Edwards, a genetic counseling program director from The University of South Carolina, enabled the inaugural Genetic Counseling Education: Connecting the Global Community conference in Manchester, United Kingdom, for educators and Chairs of genetic counseling professional institutions across the globe. As highlighted in the article by Edwards in this issue, the meetings have continued under the Transnational Alliance of Genetic Counselors (TAGC) banner.

As an attendee in Manchester, Dr. Peterson was struck by the immense value of this event in helping those who had previously only been active within their home country to appreciate the existence of a supportive global genetic counseling 
community, ready and willing to connect and share a wealth of professional experiences. The inaugural meeting generated a rich exchange of information, positive energy and a determination to not just maintain these connections but to use them to build future collaborations. This was just a first step in the development of a shared global understanding of the worldwide impact of our practice. This special issue of the Journal of Genetic Counseling was created to help provide a pulse on the current status of the global genetic counseling community. We hope that it provides another leap forward in the endeavor to understand the breadth of our community, and to continue to build valuable connections.

As program directors from the University of Michigan in the United States and Griffith University in Australia, we were first introduced to each other by Bonnie LeRoy (program director from the University of Minnesota, and Journal of Genetic Counseling Editor in Chief) at the TAGC meeting in Montreal in late 2011. It was a meeting that offered multiple opportunities to learn about the ways in which genetic counseling practitioners and educators have adapted the genetic counseling building blocks that were established in the United States to meet the unique needs of a wide variety of patients and trainees in countries across the world. In addition, we learned about novel educational tools and programs that could be implemented in our own countries. As educators and practicing genetic counselors we expressed a mutual appreciation of international networking as a potential learning opportunity that could be pivotal for those who are in the early development phase of establishing genetic counseling training programs and the profession in their region.

The journal's editorial board and we, as guest editors for this special issue, recognize that one size does not fit all when it comes to professional development in countries with differing health systems and variable sociopolitical forces. However, in theory at least, a sharing of the wealth of experience from as many countries as possible should offer relevant new strategies for those needing support in the development of the genetic counseling profession in their country. We also felt that creating an issue of the Journal of Genetic Counseling that summarized the current status of genetic counseling around the globe had the potential to provide new practice insights and identify opportunities for productive international collaborations for genetic counselors in countries with an extended history of professional activity and educational programs.

In pursuit of these goals we networked and sought submissions from regions across the globe, including Asia Pacific, Europe, the Middle East, Africa and the Americas. We anticipated receiving submissions that would provide insights into the current state of genetic counseling services and training in both well-established programs and those that were being developed. We were particularly interested in understanding the impact of each country's cultural context and health service model on the development of these programs. We were excited and a bit overwhelmed by the number of responses we received. In order to ensure the authenticity of submissions, we required the perspective of at least one reviewer with past or current clinical expertise within the relevant country or region, in addition to other expert reviewers. This special issue showcases 30 papers from 23 countries/regions: 17 brief reports, 2 professional issues papers and 11 research papers. We were pleased to receive papers from countries that expanded our awareness of local clinical genetics services (Cruz, Cuba; Erdem \& Tekșen, Turkey; Margarit et al., Chile; Micheletto et al., Brazil), and a report that gives an overview of an initiative to provide genetic service support to a country with little opportunity to develop their own services (McWalter et al., Guam). These papers highlight the impact that a few dedicated professionals can have on the provision of genetic services. They also illustrate the ways in which government-driven public health initiatives such as newborn screening, prenatal education and screening, and population-based genetic testing are creating a service vacuum that is ripe for the entry of more genetic counselors.

It was interesting to discover from the brief report from Iceland, a Nordic country that has long been at the forefront of genetic research based on extensive national DNA databases, that the challenge of increasing dedicated genetic counselor positions still exists within their health system (Stefansdottir). Regrettably, while we are aware of established genetic counseling clinical service activity in additional Nordic countries (e.g. Sweden, and Norway), we did not receive other submissions from that region. Although several research papers from the Netherlands have been published in the Journal of Genetic Counseling, we did not receive submissions for this special issue from that country or other North Eastern European countries, and therefore we will need to wait for future opportunities to better understand how genetic counseling service and educational programs are being provided in these parts of the world.

Several papers outline the critical processes employed to effectively develop guidelines for professional standards and/ or competencies (Ferrier et al., Canada; McEwen et al., Australasia; Skirton et al., UK), build educational programs (Al-Sayed et al., Saudi Arabia; Elackatt, India; Laurino \& Padilla, Philippines; Sahhar et al., Australia; Thong \& Lee, Malaysia) or implement and evaluate new services (Mendes et al., Portugal; Micheletto et al., Brazil). The growth of the profession in these parts of the world has been significant and suggests genetic counseling is now a mainstream, recognized component of health care practice. One driving force is the rise in familial cancer services; in many regions this development has provided opportunities to create and/or expand genetic counseling activity and genetic counselor roles (Gschmeidler \& Flatscher-Thoeni, Austria; Margarit et al., Chile). It might be anticipated that the scope of practice in these countries will expand with increasing recognition of the added value contributed by these practitioners. 
It was exciting to learn how the field of genetic counseling was initiated in geographically distinct parts of the world. Al-Sayed et al. from Saudi Arabia, Chen et al. from Taiwan, Cordier et al. from France, Cruz from Cuba and Sagi and Uhlmann from Israel provided overviews that highlight critical events that supported a relatively rapid development of the genetic counseling profession, and illustrate the importance of assistance provided by experienced training program directors from the Canadian and US-based programs. Papers from South America, the Caribbean, Asia Pacific, Europe and the Middle East describe how a small number of professionals manage to provide services despite challenges to professional development due to local sociopolitical context (Acosta et al., Brazil; Erdem \& Tekșen,Turkey; ; Gschmeidler \& FlatscherThoeni, Austria ; Micheletto et al., Brazil; Nishiyama et al., Japan; Zayts et al., Southeast Asia). We discovered in a paper from South Africa describing the professional practices of genetic counselors within their region, that even when there has been a substantial growth in the scope of clinical and professional services provided by genetic counselors there is still a large gap between the available resources and the needs of potential patients (Kromberg et al.). While there are clear limitations in access to genetic counseling services in each of these countries, it is exciting to learn how many different types of patient populations have access to genetic counseling.

Enthusiasm about these positive developments should be tempered by the fact that some of the papers in this edition indicate that there is limited awareness and understanding of specific genetic conditions by practitioners in training, and public knowledge about genetic counseling and the type of service provided by genetic counselors can be flawed. Research by Duan et al. exploring Fragile X knowledge in Chinese medical students, and the Maio et al. evaluation of public knowledge and perception of genetic counseling in Canada, highlight that there is still much work to be done.

Regional research papers provide a valuable new window on cultural influences on the development of genetic counseling services (Ashfaq et al., Pakistan; Duan et al., China; Ordonez et al., Chile; Micheletto et al., Brazil; Gschmeidler \& FlatscherThoeni, Austria; Zayts et al., Southeast Asia) and cultural challenges for international students studying genetic counseling in the US (Sabbadini et al.). In contrast, Alexander et al. provide insights into the value of international clinical rotation exchange programs in increasing practitioners' knowledge and comfort with these variations in cultural influence on the practice of genetic counseling.

Cultural variations are inevitable, so must be recognized and acknowledged, both in training and in practice. The process of co-editing this issue has helped us further recognize that, despite sharing identical visions of genetic counseling practice and education, there can be significant differences even between American and Australian colloquial expressions and contextual practices. While the established models of genetic counseling practice and service delivery that exist in the US, Canada, UK and Australasia are similar, they may not readily work in other countries. As the escalating application of genetic technology and information continues to generate novel tests and management options in clinical medicine, it becomes even more imperative that genetic counseling, as an essential healthcare practice, must continue to evolve to be maximally responsive to the requirements of our populations. There is a considerable need for rigorous research that will identify how cultural variations in practice are experienced by consultands and multidisciplinary health care teams. This work is critical in the development of evidence for the effectiveness of competent genetic counseling practices, and can also help identify opportunities for improvement within the global genetic counseling community.

Is the practice of genetic counseling uniform? Absolutely not. Are only accredited genetic counselors providing genetic counseling? Absolutely not. Is genetic counseling generally recognized as a crucial aspect of genetics and genomicsfocused care? The papers in this special issue demonstrate that genetic counseling as a profession is growing and is recognized by many countries as an essential service. The infrastructure is there but global development of the profession requires highly motivated individuals who will spearhead future expansion and stabilization.

In conclusion, we sincerely hope you will find this issue to be a valuable resource for further appreciation of the cultural aspects of genetic counseling service in varied populations and contexts. We also hope it will be a prompt for professionals in countries with established services to consider their opportunities to actively support others in our global professional community who are working to initiate or develop models of genetic counseling practice that are well suited to their current context. As you read through these submissions, we encourage you to think about the gaps that exist and we challenge you to both identify and create ways in which individuals and groups of professionals can bridge these gaps by building new collaborations and helping expand the reach of genetic counseling.

\section{References}

McKusick, V. A. (1997). History of medical genetics. In D. L. Rimoin, J. M. Connor, \& R. E. Pyeritz (Eds.), Emery and Rimoin's principles and practice of medical genetics (3rd ed., Vol. 1, pp. 1-30). New York: Churchill Livingstone.

Reed, S. C. (1975). A short history of genetic counseling. Social Biology, $21,332-339$.

Resta, R. G. (2006). Defining and redefining the scope and goals of genetic counseling. American Journal of Medical Genetics Part C Seminars in Medical Genetics, 142C, 269-275.

Stern, A. M. (2009). A quiet revolution: the birth of the genetic counselor at Sarah Lawrence College, 1969. Journal of Genetic Counseling, $18,1-11$ 